\title{
Maximizing Photocatalytic Efficiency of Magnetically Separable Nanoparticles for Water Disinfection by Photocatalysis
}

\author{
S. Fowler, W. Qian, M. Eastman, J. Jiao \\ Department of Physics, Portland State University, Portland OR 97207
}

Water disinfection by photocatalysis has been a topic of research and development for decades, and despite significant progress in the field, there has been very limited success industrializing this method. In order to improve the efficiency of the process, many researchers have attempted to utilize nanoparticle photocatalysts that are dispersed throughout the polluted water, rather than a using a fixed photocatalyst surface.

Although pollutant degradation efficiency is indeed increased when using the nanoparticle method, this introduces the technical difficulty of retrieving the nanoparticles for reuse. One approach to solving this problem is the fabrication of coreshell nanoparticles, which have a magnetic core and a photocatalytic outer shell. However, the magnetic core has been shown to have a detrimental effect on reaction rate [2]. Thus, our goal is to mitigate this effect by including an insulating barrier layer between the core and shell. Currently, the work is in progress and this abstract presents magnetic $\mathrm{Fe}_{3} \mathrm{O}_{4}$ particles synthesized by a solvothermal process, which we believe are suitable for this purpose. A Raman spectrum of the synthesized particles was obtained (Figure 1) and a comparison with previously published results [1] verifies that the produced material is indeed $\mathrm{Fe}_{3} \mathrm{O}_{4}$.

In order to maximize the photocatalytic degradation efficiency, several factors must be taken into consideration. First, the particle must show superparamagnetic properties, so that it can be separated magnetically, but without detrimental agglomeration. The magnetic susceptibility measurements, shown in Figure 2, indicate that there is no residual magnetization of the particles, and hence they are indeed superparamagnetic. Second, the core particle should be a single crystal so that there is no cancelation of dipole moments as is the case within a polycrystalline structure, thus increasing the magnetic susceptibility to volume ratio. The TEM micrograph in Figure 3 shows that synthesized particles are in fact single crystals. And third, since the next step is to create an insulating layer onto the outside of the magnetite core particle, it is necessary that the core particles are monodisperse and have a narrow size distribution in order to create a symmetric and stable shell. These characteristics are displayed in the TEM micrograph in Figure 4. For these, we believe that nanoparticles characterized above are good candidates for the core of a multi-layer magnetically separable photocatalyst.

References:

[1]. J. Raman Spectrosc., Vol. 34. (2003), pp.845-852.

[2]. Applied Catalysis B: Environmental, Vol. 100, No. 1-2. (October 2010), pp. 338-345.

[4]. This research is supported by a private donation to the Portland State University

Foundation. The material characterization was carried out at the PSU Center for Electcron Microscopy and Nanofabrication. 


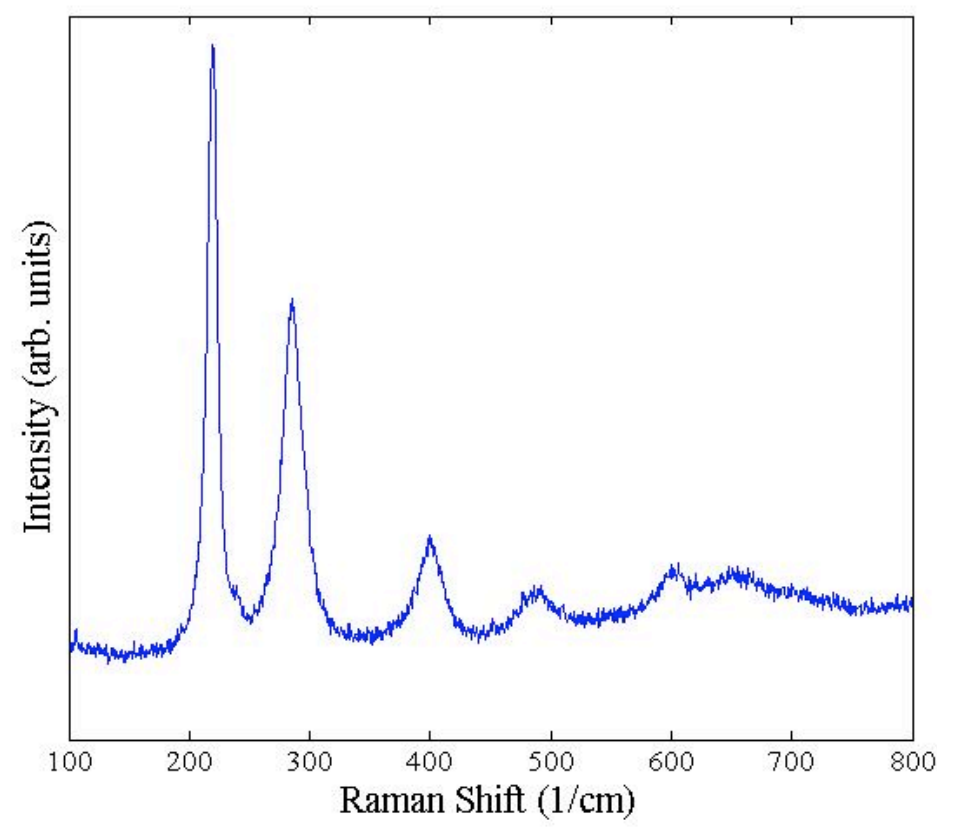

Figure 1: Raman spectrum from excitation by 532nm laser, showing the characteristic Raman shifts of magnetite.

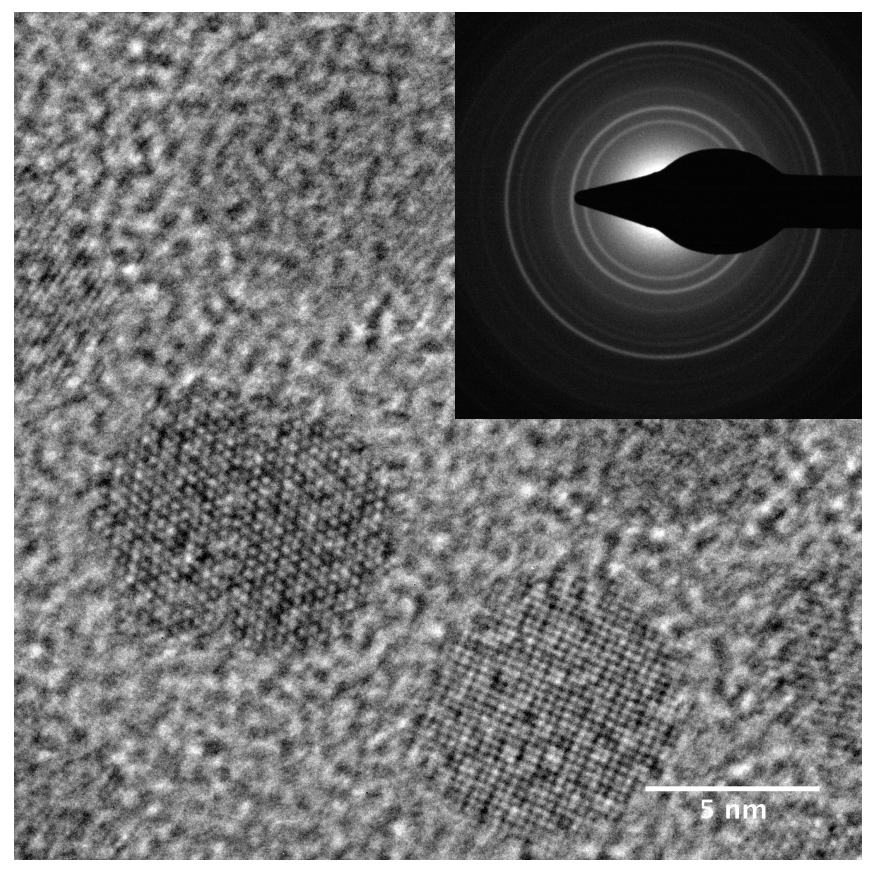

Figure 3: TEM micrograph indicates the $\mathrm{Fe}_{3} \mathrm{O}_{4}$ nanoparticles grown by solvolthermal process are uniform, and single-cyrstalline with a diameter of $7 \mathrm{~nm}$. The inset shows the SAED of the particles on amorphous carbon film.

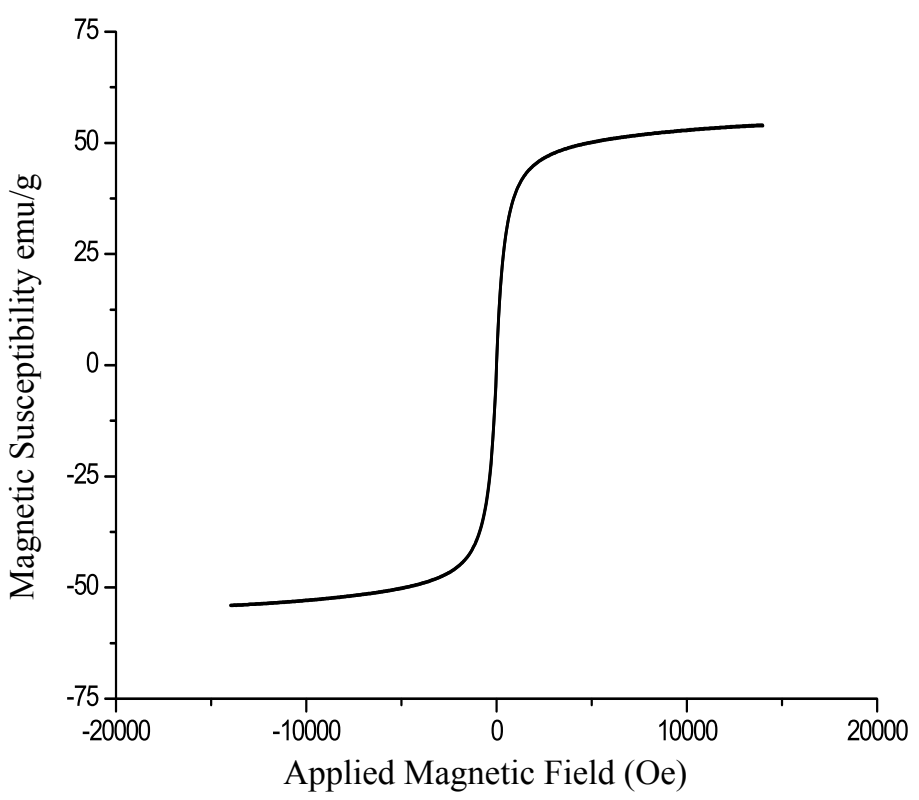

Figure 2: Magnetic susceptibility measurement showing the superparamagnetic property of the $\mathrm{Fe}_{3} \mathrm{O}_{4}$ particles synthesized by this method.

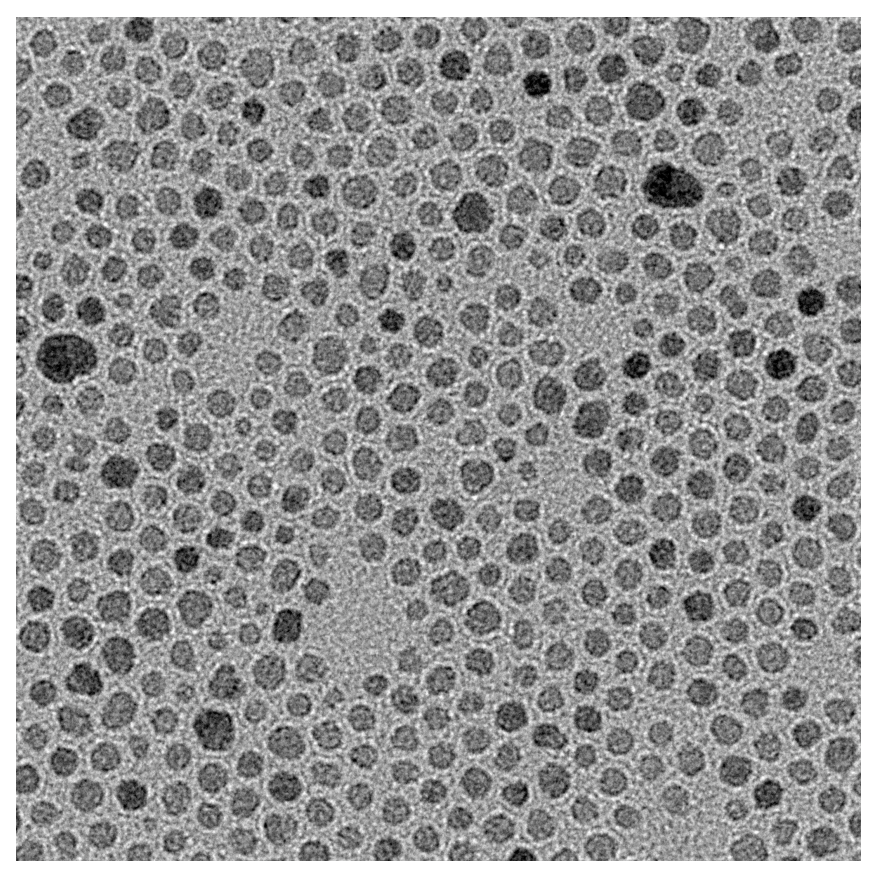

Figure 4: Low magnification TEM image showing monodisperse particles with narrow size distibution. 